\title{
Rétinoïdes et bases moléculaires des malformations cardiaques congénitales
}

I.es malformations cardiaques constituent plus de $25 \%$ des malformations congénitales et représentent ainsi la classe la plus importante de malformations congénitales chè. l'humain. Cies malformations sont multifactorielles et impliquent des causes génét iques et liées à l'environnement. Par exemple, la carence maternelle en vitamine A provoque plusieurs malformations congénitales incluant des malformations cardiaques majeures associées à une létalité embryonnaire. I'acide rétinoïque (9-cis acide rétinoïque et trans acide rétinoïque), un métabolite de la vitamine $\mathrm{A}$, possède plusieurs effets tératogènes chevauchant les effets de la carence matemelle en vitamine A. Ce spectre d'action superposé suggère que l'acide rétinoïque est un morphogène, c'est-à-dire qu'il véhicule une information quant à la position durant le développement. Dans le cas du coeur embryonnaire, des expériences chez le poulet et le poisson-zèbre indiquent que l'acide rétinoïque est probablement l'un des signaux locaux impliqués dans la polarité du tube cardiaque et la différenciation des compartiments (chambres) cardiaques. Cependant, la base moléculaire du rôle de l'acide rétinoïque dans la morphogenèse cardiaque, incluant les effecteurs intracellulaires et les gènes cibles, était inconnue. Trois papiers parus récemment |1-3] fournissent les premières domnées sur les effecteurs intracellulaires de l'acide rétinoïque dans le tissu cardiaque.

L.es effets de l'acide rétinoïque sont transduits par deux groupes de récepteurs de la superfamille des récepteurs nucléaires, soit les RAR $($ RAR $\alpha, \beta, \gamma)$ qui sont activés par les deux isomères de l'acide rétinoïque et les $\operatorname{RXR}(\operatorname{RXR} \alpha, \beta, \gamma)$ dont le ligand est le 9-ris acide rétinoïque. Chacune de ces isoformes est soumi- complexité de la réponse à l'acide rétinoíque est amplifiée par la formation d'hétérodimères RAR:RXR qui représenteraient l'entité physiologique de réponse à l'acide rétinoïque.

L'inactivation du gène codant pour $\operatorname{RXR} \alpha[1,2]$ provoque des malformations cardiaques incompatibles avec la vie puisque les embryons meurent entre les jours embryonnaires 10,5 à 16,5 , probablement du fait d'une insuffisance cardiaque majeure qui empêche le coeur de subvenir aux besoins d'apport sanguin de l'embryon. L'étude histologique du coeur des souris mutantes dénote un phénotype de coeur spongieux, caractérisé par une désorganisation de la trabeculae du ventricule et une hypoplasie ventriculaire. Plusieurs malformations du septum ventriculaire sont aussi observées. Ciependant, les oreillettes ne sont que faiblement atteintes. Ces malformations sont très similaires, sinon identiques, à celles observées sur la progéniture de mères déficientes en vitamine A. En dépit du fait que l'inactivation de RAR $\gamma, \operatorname{RAR} \alpha$ ou RAR $\beta 2$ ne provoque pas de malformations cardiaques, les doubles inactivations RAR $\alpha \gamma$ ou $\alpha \beta 2$ domment un phénotype de coeur spongieux avec une pénétrance comparable à celle de l'inactivation de $\operatorname{RXR} \alpha$ [3]. Ces résultats suggèrent une convergence de la signalisation dépendante des rétinoïdes et une redondance fonctionnelle entre les différents membres de la famille RAR. Ie plus, ces résultats seraient cohérents avec l'hypothèse que l'hétérodimère RXR:RAR est le médiateur physiologique de la réponse à l'acide rétinoïque.

Si l'on commait maintenant les effecteurs intracellulaires de l'acide rétinoïque lors de la cardiogenèse, les gènes cibles doivent encore être identifiés. Il faudra également déterminer les cellules cibles de l'action des rétinoïdes, d'autant plus que la présence de $\operatorname{RXR} \alpha$ dans les cardiomyocytes est controversée. L.es candidats pour les gènes cibles sont nombreux; le gène du TGF $\beta$ est particulièrement attrayant puisque l'inactivation de ce gène produit un phénotype cardiaque qui ressemble beaucoup au phénotype du coeur spongieux obtenu par inactivation du gène $\mathrm{RXR} \alpha$ ou des gènes RAR $\alpha \gamma$ et $\alpha \beta 2$ [4]. I.e croisement entre des souris TGF $\beta+/$ - et des souris $\mathrm{RXR} \alpha+/$ - pourrait permettre de vérifier s'il y a synergie ou redondance entre le TGF $\beta$ et l'acide rétinoïque dans le développement cardiaque. De plus, des expériences in vitro d'explant ou de cultures provenant de coeurs embryomnaires de souris mutantes pourraient permettre de déterminer si le phénotype est autonome ou s'il est induit par l'anomalie d'une structure embryomnaire avoisinante, et de caractériser l'état de différenciation du coeur. C.es informations pourraient permettre d'établir la cascade des événements menant à la formation de la trabeculae et du septum ventriculaires ainsi que d'identifier les mécanismes menant à la polarité antéro-postérieure (ventricule-oreillette) du myocarde qui dépendrait de l'action des rétinoïdes.

D.D.

M.N.

1. Kastner I', Cirondona JM, Mark $M$, (jansmullerA, I e Meur M, Decimo) 1), Vonesch Jl. Dolle P', (hambon P. (ienetic analysis of RXRo developmental function: convergence of $R \times R$ and RAR signaling palhways in heart and eye morphogenesis. (ell 1994; 78 : 987-1003.

2. Sucov HM, Dyson E, (iumeringer (CI, Price I, (Chien KR, Evans RM. RXR a mutant mice establish a genetic basis for vitamin A signaling in heart morphogenesis. (ienes Dea 1994; 8 : 1007-18.

3. Mendelshon (', Mark M, Dolli I', Dierich A, (iaub) MP, Krust A, lampron (;, Chambon P. Retinoic acid receptor $\beta 2$ (RAR $\beta 2)$ null mutant mice appear normal. Devolopment 1994; 120 : 2749-71. 4. Nemer M. Révaluation du rôle de T( $F F /$ dans le développement du corur. méderine/science 1994 ; 